\title{
Melatonin for pre-medication in children: a systematic review
}

\author{
Katie Mellor ${ }^{1}$, Diana Papaioannou ${ }^{1}$, Anna Thomason ${ }^{1}$, Robert Bolt ${ }^{2 *}$, Chris Evans ${ }^{3}$, Matthew Wilson ${ }^{1}$ and \\ Chris Deery ${ }^{2}$
}

\begin{abstract}
Background: Melatonin's effectiveness as an anxiolytic medication has been confirmed in adults; however, its efficacy in a paediatric population is unclear. A number of small studies have assessed its use in children as a pre-operative anxiolytic, with conflicting results.

Methods: We undertook a systematic review of pre-operative melatonin use in children. Four databases (MEDLINE, Embase, the Cochrane Central Register of Controlled Trials and Web of Science), and 'ClinicalTrials.gov' were searched for ongoing and completed clinical trials of relevance. Citation tracking reference lists and relevant articles were also accessed. The review was unrestricted by comparator or outcomes. Eleven studies were judged eligible for inclusion. There were high levels of heterogeneity in melatonin administration (in terms of dose and timing). Variable outcomes were reported and included: anxiety; anaesthetic success; analgesia; sedation; post-operative recovery; and safety. Outcomes were not always assessed with the same measures.
\end{abstract}

Results: Evidence to support melatonin's anxiolytic properties in this setting is conflicting. Melatonin was associated with reduced sedative effects, post-operative excitement and improved emergence behaviour, compared to comparator drugs. One study reported the benefit of melatonin use on sleep disturbance at two weeks post-surgery. No adverse safety events were identified to be significantly associated with melatonin, affirming its excellent safety profile.

Conclusion: Despite potential advantages, including improved emergence behaviour, based on current evidence we cannot confirm whether melatonin is non-inferior to current "usual care" pre-medications. Further consideration of melatonin as an anxiolytic pre-medication in paediatric surgery is needed.

Keywords: Melatonin, Children, Pre-medication, Surgery, Anxiety

\section{Background}

Melatonin is a natural sleep promoting neurohormone synthesised within the pineal gland. Aside from regulation of circadian rhythm, melatonin's physiological functions include antioxidant, oncostatic, anti-inflammatory and anticonvulsant effects [1]. Melatonin can be produced synthetically. European Medicines Agency

*Correspondence: r.bolt@sheffield.ac.uk

2 School of Clinical Dentistry, University of Sheffield, Sheffield, UK

Full list of author information is available at the end of the article licensed tablets ( 2 mg Circadin ${ }^{\circledR}$, UK and 3 mg Bio Melatonin, Hungary), and a $1 \mathrm{mg} / \mathrm{ml}$ oral solution exist (Colonis Pharma). Unlicensed liquid formulations are also available in the UK (Kidmel ${ }^{\circledR}$ \& Kidnaps ${ }^{\circledR}$, Special Products Limited, UK), as well as unlicensed 2-3 mg generic capsule formulations [2].

Melatonin is used in children and neonates to manage a number of conditions, including sleep and seizure disorders and neonatal sepsis. Melatonin has also been evaluated for its use as a pre-operative anxiolytic and has promising potential due to its reduced sedative effect 
compared to other anxiolytics. Melatonin's anxiolytic properties are considered to be a consequence of its facilitatory role on $\gamma$-aminobutyric acid (GABA) transmission [3]. Although the effectiveness of melatonin as an anxiolytic pre-medication in adults has been confirmed through multiple clinical trials [4-10] and systematic reviews [11-13], its usefulness in a paediatric pre-operative setting is less certain. The existing literature has described an excellent safety profile; melatonin has no known major side effects and is well-tolerated [14].

Standard anxiolytic pre-medications in the paediatric setting include benzodiazepines, alpha2 agonists (clonidine or dexmedetomidine), and $\mathrm{H} 1$ antihistaminics [15]. Although effective, these drugs are associated with an increased sedative effect that may lengthen post-anaesthetic recovery. Melatonin offers a number of potential advantages, including ambulant rather than bed transfer to theatre, reduced post-operative sedation \& sleep disturbance, faster recovery, improved post-operative analgesia, and avoidance of respiratory depression [16, 17]. In addition, some melatonin formulations may offer greater taste acceptance compared to the bitter flavour of conventional pre-medications, which could potentially improve compliance in a paediatric population. A number of small clinical trials have been conducted to assess melatonin pre-medication in the paediatric setting [1828 ], although results are conflicting. Given the potential benefits melatonin has over alternative pre-medications, there is a need to determine whether there is an evidence base for its anxiolytic function in children.

In 2014, Andersen et al. [11] published a systematic review and meta-analysis of the efficacy and safety of peri-operative melatonin, finding a significant reduction in post-operative pain and pre-operative anxiety. The review drew from a general adult \& child population, and since publication there has been a number of more recent randomised controlled trials conducted in a specifically paediatric population.

The primary aim of this systematic review is to determine the current evidence for the use of melatonin as a pre-operative anxiolytic in children.

\section{Methods}

A protocol for this systematic review is available on PROSPERO (registration: CRD42018098940). The review has been conducted and reported according to PRISMA guidelines [29]. Randomised controlled trials (RCTs) were included with no restriction on comparator, outcomes, randomisation generation, blinding, publication date or language. The population was limited to children (aged 0-18 years). Studies evaluating any surgical intervention were considered eligible. Studies evaluating medical diagnostic procedures were excluded. No restriction was placed on melatonin formulation or dosage, trial comparator, or outcome. Four electronic databases were searched including MEDLINE, Embase, the Cochrane Central Register of Controlled Trials and Web of Science. 'ClinicalTrials.gov' was searched for ongoing and completed clinical trials. Backward citation tracking of reference lists, and forward citation tracking of relevant articles were also used. The search strategy is presented in Additional file 1.

Literature search exports were de-duplicated by KM using Elsevier Mendeley Desktop software [30], and confirmed by the built in 'Check for Duplicates' function. Two independent reviewers (KM and AT) considered the title and abstract of each study, excluding those not relevant. KM obtained the full texts of all studies that appeared eligible. KM and AT independently identified those studies for final inclusion. Where eligibility queries arose, guidance from a senior reviewer (DP) was sought.

A tailored data extraction form in Microsoft Excel was used to extract data. This was undertaken by KM and confirmed by AT. Data collected included: publication details, study design and characteristics; surgery details; anaesthesia details; intervention details (including melatonin dose, formulation, route of administration, and timing given); comparator details; any outcome measures and adverse events reported. The latest version (October 2018) of the revised Cochrane risk-of-bias tool for randomized trials (RoB 2.0) was used to assess bias of included studies [31]. This was undertaken independently by KM and AT. Any discrepancies were resolved by discussion with DP. Authors were contacted for clarification where information was omitted from the publication.

Summary measures are reported in a narrative synthesis. A meta-analysis was not appropriate in this study due to the small body of existing trials, and heterogeneity of the data. Findings with a P-value of $<0.05$ were considered to be significant and are described as such.

\section{Results}

Electronic database searches retrieved 1148 results. Three additional results were obtained through citation tracking and screening reference lists. 920 results remained following deduplication. The abstracts of 61 articles were screened, of which 16 full text articles were assessed. Five of these were deemed ineligible due to either study design or population (i.e. did not investigate children), leaving eleven articles for inclusion in the review [18-28] (Fig. 1).

\section{Study characteristics}

Included studies were published between 2005 and 2018, and were conducted in Italy, Iran, Turkey, USA, Egypt and India. Sample sizes ranged from 23 to 148 children 


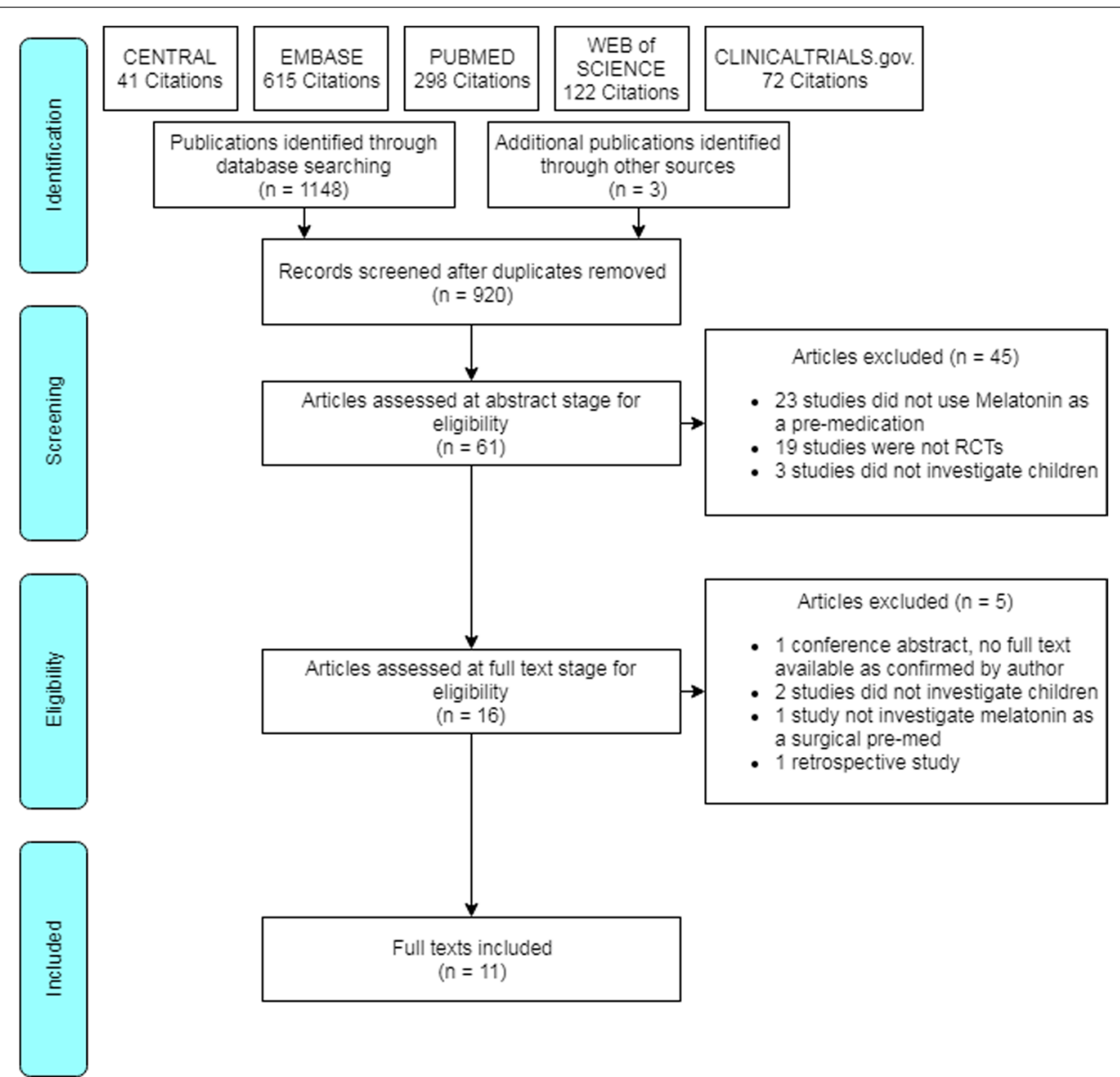

Fig. 1 PRISMA flow chart

and population age ranged from 1 to 15 years. Surgical populations included dental $[18,19,28]$, adeno-tonsillectomy [24], oesophageal dilatation procedures [26], and other forms of minor elective surgery [21-23, 25, 27]. General anaesthesia was used in eight studies [19-24, 26, 27]. One study did not specify type of anaesthesia; the authors confirmed that both local and general anaesthesia were used [25]. Two studies used sedation without local anaesthesia $[18,28]$. Three studies restricted inclusion criteria to an anxious population $[18,19,28]$, defined as having negative/uncooperative behaviour according to the Frankl Behavioural Scale (FBS) [32]. Full study details are summarised in Table 1.

\section{Comparators}

Ten studies compared melatonin to midazolam [18, 19, 21-28], either directly or amongst other arms including placebos $[18,19,24,25,27]$ and dexmedetomidine [26]. One study compared melatonin with clonidine alone [20]. One study compared melatonin to ketamine, placebo, and a combination of melatonin and ketamine in half doses [24].

\section{Dose and formulation of melatonin}

Three studies used Melamil ${ }^{\circledR}$ oral drops, [20-22] one Melatonina ${ }^{\circledR}$ tablets [18], and the remaining studies did not specify formulation or trade name. All but two studies provided manufacturer information [18-23, 25, 27, 28], which included companies in Italy, Poland, the USA and India. One study [25] noted that the Melatonin was obtained online (Life Extension pharmaceuticals). Two studies did not report manufacturer information [24, 26]. Melatonin preparation varied. Five studies mixed the active ingredient with water (ranging from $3-10 \mathrm{ml}$ ) [18, $19,21,22,28]$, and three gave melatonin together with oral paracetamol $[24,25,27]$. One study used melatonin syrup, and therefore no preparation was required [25]. One study did not specify preparation [20]. All studies administered the medication orally. Dosing ranged from $0.05 \mathrm{mg} / \mathrm{kg}$ to $0.75 \mathrm{mg} / \mathrm{kg}$, and two studies capped dosage at $20 \mathrm{mg}[21,22]$. Three studies assessed melatonin 


\begin{tabular}{|c|c|c|c|c|c|c|c|c|c|c|c|}
\hline 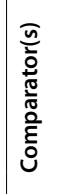 & 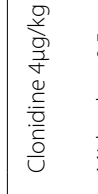 & 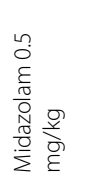 & 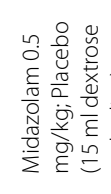 & 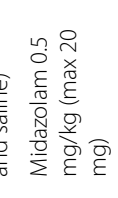 & 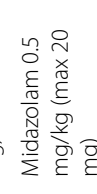 & 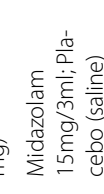 & 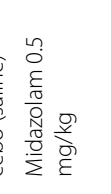 & 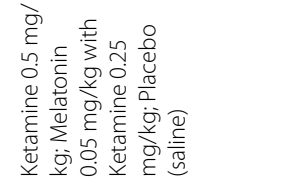 & 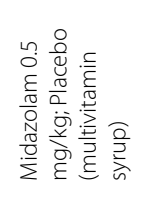 & 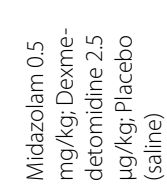 & 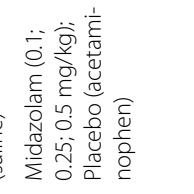 \\
\hline 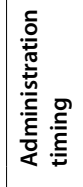 & 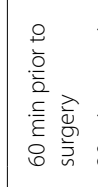 & 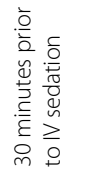 & 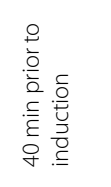 & 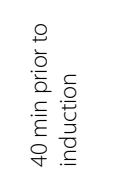 & 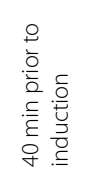 & 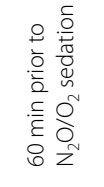 & 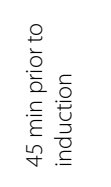 & 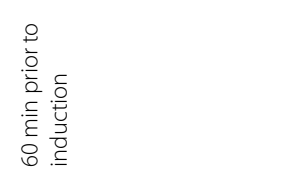 & 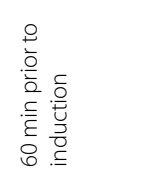 & 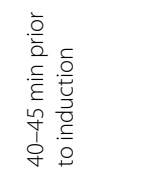 & 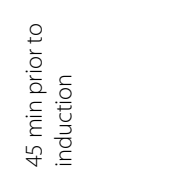 \\
\hline 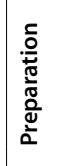 & 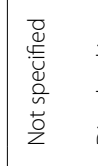 & 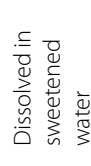 & 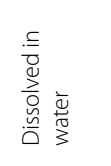 & 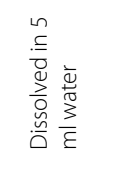 & 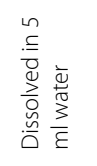 & 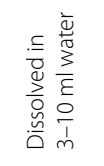 & 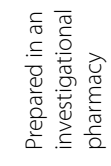 & 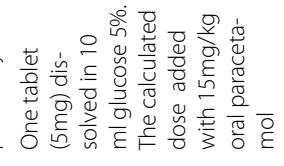 & 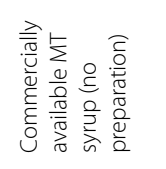 & 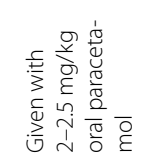 & 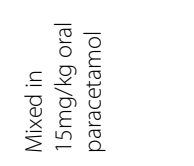 \\
\hline 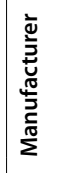 & 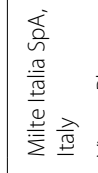 & 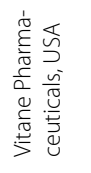 & 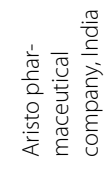 & 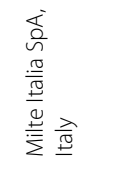 & 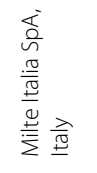 & 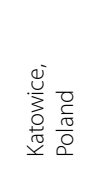 & 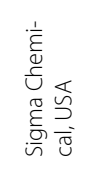 & 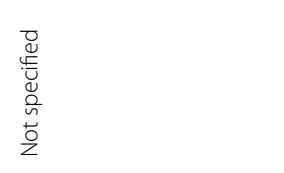 & 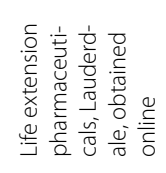 & 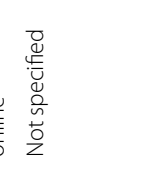 & 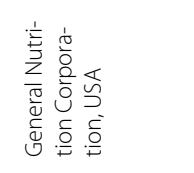 \\
\hline 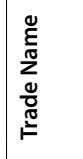 & 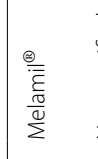 & 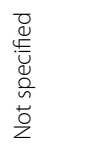 & 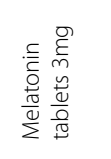 & 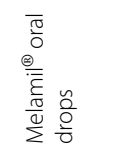 & 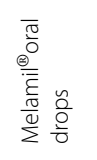 & 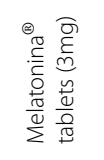 & 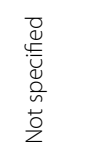 & 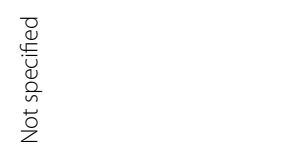 & 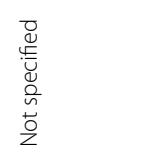 & 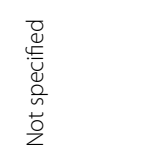 & 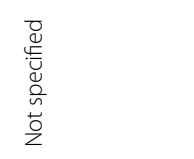 \\
\hline 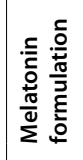 & $\begin{array}{l}\bar{\xi} \\
\text { छे } \\
\text { है }\end{array}$ & 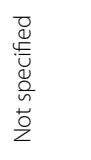 & $\begin{array}{c}\bar{\varepsilon} \\
\stackrel{\underline{n}}{ }\end{array}$ & 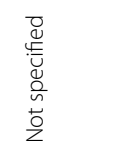 & 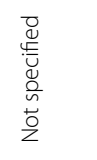 & 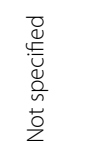 & 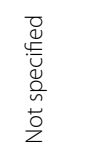 & 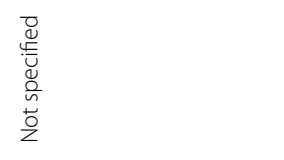 & $\begin{array}{l}\bar{\xi} \\
\text { छे } \\
\text { ह }\end{array}$ & 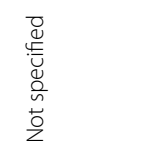 & 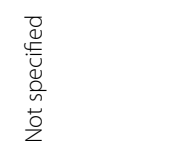 \\
\hline 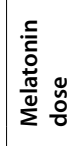 & $\begin{array}{l}\text { g. } \\
\text { gे } \\
\text { हn } \\
\text { o }\end{array}$ & $\begin{array}{l}\text { शे } \\
\text { है } \\
\text { है } \\
\text { nू }\end{array}$ & $\begin{array}{l}\text { g) } \\
\text { है } \\
\text { हn } \\
0\end{array}$ & 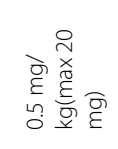 & 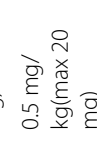 & 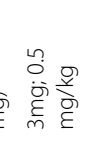 & 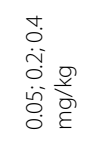 & $\frac{\text { g) }}{\frac{\text { v }}{\varepsilon}}$ & 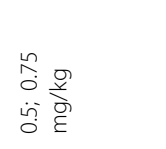 & $\frac{\text { gे }}{\stackrel{5}{\varepsilon}}$ & 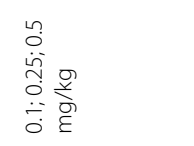 \\
\hline 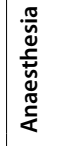 & $\mathbb{J}$ & 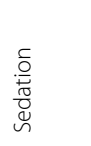 & $\mathbb{J}$ & $\mathbb{J}$ & $\mathbb{J}$ & 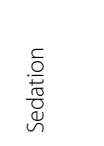 & $\mathbb{J}$ & $\mathbb{\Xi}$ & 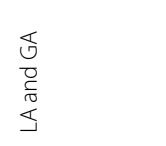 & 芯 & 芯 \\
\hline 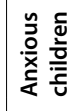 & $\stackrel{2}{z}$ & $\stackrel{\check{\varpi}}{\check{\nu}}$ & $\stackrel{\breve{巛}}{\check{\nu}}$ & 足 & z & 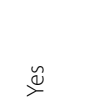 & $\stackrel{\circ}{z}$ & $\stackrel{\circ}{z}$ & $\stackrel{\circ}{z}$ & \& & $\stackrel{\circ}{z}$ \\
\hline$\stackrel{\breve{\Sigma}}{\check{\Sigma}}$ & $\stackrel{\circ}{\stackrel{ }{K}}$ & $\stackrel{\circ}{\check{L}}$ & 㣽 & ণ্তু & $\frac{\circ}{\stackrel{+}{0}}$ & $\stackrel{\stackrel{一}{m}}{m}$ & $\underset{\infty}{\stackrel{\infty}{~}}$ & $\begin{array}{l}\stackrel{\circ}{\circ} \\
\stackrel{\leftrightarrow}{0}\end{array}$ & $\frac{g}{i t}$ & 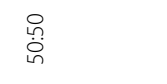 & $\underset{\tilde{N}}{\tilde{N}}$ \\
\hline 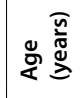 & $\stackrel{n}{\check{1}}$ & $\stackrel{i}{\sim}$ & $\begin{array}{l}\dot{0} \\
\dot{m}\end{array}$ & $\frac{\vec{J}}{\dot{L}}$ & $\begin{array}{l}\frac{J}{1} \\
\infty\end{array}$ & $\begin{array}{l}\infty \\
\dot{m}\end{array}$ & $\begin{array}{l}\infty \\
\dot{\sim}\end{array}$ & $\begin{array}{l}\dot{0} \\
\dot{m}\end{array}$ & $\stackrel{n}{\llcorner}$ & $\begin{array}{l}a \\
\dot{m}\end{array}$ & $\stackrel{n}{\sim}$ \\
\hline 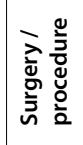 & 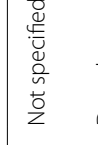 & 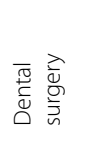 & 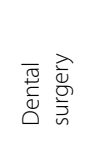 & 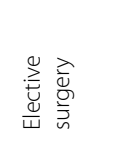 & 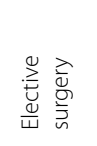 & 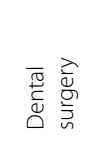 & 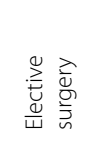 & 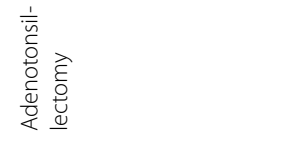 & 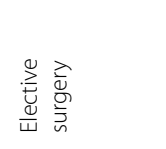 & 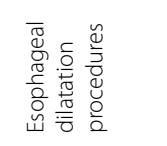 & 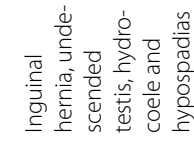 \\
\hline$=$ & $\infty$ & $\ddot{\sim}$ & $\tilde{m}$ & $\alpha$ & $\infty$ & 8 & $\stackrel{\infty}{+}$ & 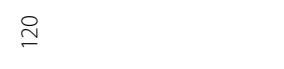 & $\stackrel{8}{-}$ & $\stackrel{8}{ }$ & $\stackrel{n}{\circ}$ \\
\hline 童 & 를 & 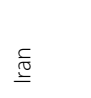 & 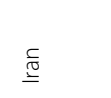 & $\overrightarrow{\text { 를 }}$ & 츹 & 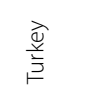 & $\stackrel{\overleftarrow{s}}{3}$ & 营 & 登 & 站 & $\stackrel{\overleftarrow{s}}{3}$ \\
\hline 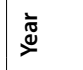 & $\stackrel{m}{i}^{m}$ & $\stackrel{\infty}{\grave{\sim}}$ & $\stackrel{\infty}{\grave{i}}$ & $\stackrel{0}{\circ}$ & $\bar{i}$ & 怘 & ठ্ণे & $\stackrel{n}{\grave{d}}$ & $\stackrel{0}{\grave{2}}$ & $\overline{\bar{i}}$ & 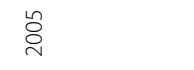 \\
\hline 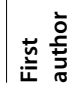 & 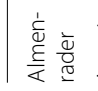 & $\begin{array}{l}\text { 高 } \\
\text { 尊 }\end{array}$ & 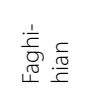 & 㟢 & $\begin{array}{l}\frac{1}{\bar{\alpha}} \cdot \overline{\bar{\nu}} \\
\underline{\underline{\underline{N}}}\end{array}$ & 鸢 & 高 & 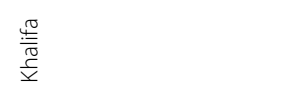 & 訔 & 㝘. N & 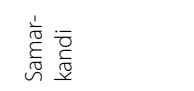 \\
\hline
\end{tabular}


effect using a dose range; Kain et al. trialled 0.05, 0.2 and $0.4 \mathrm{mg} / \mathrm{kg}$ [23], Kurdi et al. trialled 0.5 and $0.75 \mathrm{mg} / \mathrm{kg}$ [25] and Samarkandi et al. trialled $0.1,0.25$ and $0.5 \mathrm{mg} / \mathrm{kg}$ [27]. The timing of melatonin administration varied from 30-60 min prior to induction of anaesthesia. Intervention details are summarised in Table 1.

\section{Outcome measures}

Five studies explicitly stated a primary outcome measure. These included success of steal induction (inhalational induction in a sleeping child) [20], effect on propofol requirements [21], pre-operative anxiety [22, 23], and anxiolysis, sedation, maintenance of cognition \& psychomotor skills [25]. Additional reported outcomes included analgesia [19, 27], emergence behaviour [24, 26], duration of recovery [19, 20, 27], success of anaesthetic induction $[19,22,23,25]$, and adverse events $[18,20,28]$. Reported outcomes are summarised in Table 2.

\section{Risk of bias assessment}

Bias was assessed in all RCTs as per the Cochrane risk of bias assessment tool v2.0 [31]. Methodological quality assessed included selection, performance detection, attrition and reporting bias (Table 3). All studies used a method of random sequence allocation; methods included computer-generated lists and random number tables. Methods of allocation concealment included the use of sealed envelopes and central computer generated allocation [19-27]. Ten studies were double blinded (both participants and outcome assessors blinded to allocation) $[18,19,21-28]$; however only four studies specified that the melatonin administrator was also blinded [21-23, 27]. One study was single blinded to outcome assessors [20]. Two studies did not state whether outcome data was available for all randomised participants [19, 21].

Eight studies were designed to test an a priori proposed difference in treatment effect between the study interventions i.e. they were designed as superiority trials. In general, sample sizes were based on large treatment effects. Two studies provided complete details on sample size calculation, including an explanation for the selected difference in treatment effect $[20,23]$. Six studies, although providing detail on sample size parameters, did not justify why they had selected a particular treatment effect $[18,22,24-27]$. Three studies failed to provide any details on how the sample size had been calculated $[19,21,28]$.

\section{Outcomes Explored}

\section{Anxiety}

Pre-operative anxiety was assessed in four studies (total 433 children) [22, 23, 25, 27]. Tabular data was available in all studies, with three also presenting graphical data
$[23,25,27]$. No studies reported significantly different baseline anxiety levels between trial arms.

All studies used the Modified Yale Pre-operative Anxiety Scale (mYPAS) to assess pre-operative anxiety [33]. The Spielberger State-Trait Anxiety Inventory for Children (STAI-C) [34] was used in one study to assess child anxiety the day before surgery [22]. Assessment time points varied between studies and were either specified by event, e.g. 'day before surgery' [22]; 'before premedication' [25, 27]; 'in the pre-operative room' [22]; 'separation from parents' $[25,27]$; or numerically, e.g. ' 45 minutes prior to induction'; '10, 30, 45, 60 minutes following pre-medication'; and ' 10 minutes post-operative' $[22,23,25,27]$.

There is conflicting evidence for the use of melatonin as a pre-operative anxiolytic in children. Kurdi et al. supported the use of melatonin $(0.5$ and $0.75 \mathrm{mg} / \mathrm{kg})$ to decrease pre-operative anxiety, with the higher dose $(0.75 \mathrm{mg} / \mathrm{kg})$ reported to be most effective [25]. Impellizzeri et al. and Samarkandi et al. concluded melatonin $(0.25$ and $0.5 \mathrm{mg} / \mathrm{kg})$ to be equally as effective as comparators in reducing anxiety [22, 27]. Kain et al. reported that children who received melatonin at a range of lower dosages $(0.05,0.2$ and $0.4 \mathrm{mg} / \mathrm{kg})$ were significantly more anxious compared to comparator, with no significant difference between doses [23]. In addition, all four studies assessing anxiety related outcomes, did not study a specifically anxious population, thereby potentially diluting any observable effects as an anxiolytic compared to either active or placebo control.

Two studies assessed parental anxiety prior to surgery $[22,23]$ using the STAI [34]. One study assessed anxiety of mothers only [22]. In both studies parental anxiety did not differ between the two arms. One study did identify a statistically significant correlation between mother's and child's anxiety in both trial arms [22]. Where parental anxiety was measured at different time points during the preoperative period, there was a significant increase in anxiety at later time points such as at separation from the child in both arms, with no significant association to melatonin dose or comparator [23].

\section{Anaesthetic induction}

Evidence for the effect of melatonin on induction compliance (total 460 children), was conflicting [19, 22, 23, 25]. One study found no significant difference between melatonin and midazolam against the Induction Compliance Checklist (ICC) [22], another concluding significantly lower ICC in children pre-medicated with melatonin compared to midazolam (50 vs 73\%) [23]. In terms of IV access, Faghihian et al. reported that midazolam was superior (statistically significant) to melatonin on ease of IV access, and melatonin was not 
statistically different to placebo [19]. On the contrary Kurdi et al. reported greatest venepuncture compliance at the highest dose of melatonin, although results were not statistically significant [25].

There was no significant difference between melatonin compared to clonidine for the performance of steal induction (clonidine was effective in 13\% more children), and the efficacy of melatonin was found to be dependent on the time of day administered. No age-dependent effect of melatonin was observed [20]. Gitto et al. investigated the effect of melatonin pre-medication, compared to midazolam, on propofol infusion requirements. The study concluded that melatonin significantly reduced the overall dosage of propofol infusion [21].

\section{Sedation}

Melatonin's sedative effect prior to anaesthesia was assessed in five studies (total 407 children). All studies used different measures of sedation success including a unreferenced Sedation Scale [19], the Ramsay Sedation Scale (RSS) [18, 35], an Observers Sedation Scale (OSS) [25, 36], a Houpt Sedation Rating Scale [28, 37] and the University of Michigan Sedation Scale (UMSS) [21, 38]. Four studies reported that melatonin did not contribute towards sedation prior to anaesthesia, was similar to placebo, and was inferior to comparators $[18,19,25$, 28]. Three/four studies investigated a specifically anxious population (according to the Frankl Behavioural Scale (FBS)) $[18,19,28]$. Gitto et al. reported that patients who had received melatonin were equally as sedated as those who had received midazolam [21]. Based on these five studies there is some evidence for melatonin's reduced sedative effect in this setting.

\section{Analgesia}

Two studies reported pain associated outcomes. Faghihian et al. found melatonin to reduce post-operative pain compared to placebo (quantified as analgesic requirements, of any modality, to discharge) [19]. The effect of melatonin compared to midazolam on need for analgesics is unclear, as the tabular data conflicts with the text. Samarkandi et al. reported melatonin to be superior to midazolam in reducing post-operative excitement (assessed using the modified pain/discomfort scale at $10 \mathrm{~min}$ post-operative). As the authors state, this scale does not differentiate between pain and excitement. In addition, all children received a caudal block and paracetamol which confounds evidence to support any analgesic benefit [27]. Based on these studies there is limited clinical evidence to support the analgesic effect of melatonin.

\section{Recovery}

Emergence behaviour was reported for 368 children. Kain et al. reported a statistically significant reduction in emergence delirium with melatonin compared to midazolam, as assessed by the Keegan scale [23, 39]. Two studies reported significant reductions in emergence agitation compared to placebo, with the reduction being similar to other comparators $[24,26]$.

Two studies evaluated post-operative recovery using the Aldrete Scale [40, 41]; both reported no significant difference between melatonin and comparator [21, 27]. Only one study investigated a longer-term follow up outcome post-discharge. Samarkandi et al. assessed sleep disturbance two weeks post-operatively using the Post Hospitalisation Behaviour Questionnaire (PHBQ) [42], reporting that melatonin pre-medication was associated with a significantly lower incidence of sleep disturbance compared to midazolam [27].

Recovery duration was assessed in three studies (total 324 children) and was conflicting. Almenrader et al. reported no significant difference between melatonin and clonidine in time to discharge [20]. Faghihian et al. reported that patients who had received melatonin had a significantly longer recovery (defined as time to discharge, decided by the anaesthetist based on the modified Aldrete criteria) than those who had received midazolam [19]. Samarkandi et al. reported that melatonin was associated with a faster recovery compared to midazolam (defined as scoring eight on the modified Aldrete scale), although this result was not statistically significant. Samarkandi also noted a trend in increased midazolam dose and protracted recovery duration. This trend was not present for melatonin [27]. There is overall conflicting evidence for the effect of melatonin pre-medication on recovery outcomes.

\section{Safety profile}

Adverse events (AEs) were reported in three studies [18, $20,28]$, and three further studies explicitly stated that there were no associated AEs [25-27]. Where AEs were not mentioned in the publication the authors were contacted, with three further authors confirming that no AEs were recorded throughout the duration of the study [21, $22,28]$. AEs were rarely associated with melatonin use. Two studies recorded post-operative nausea and vomiting, cough and hiccough within both melatonin and comparator groups $[18,20]$. One study reported a significantly lower incidence of nausea and vomiting, tremors and dizziness in children pre-medicated with melatonin compared to midazolam [28]. In further support of melatonin's safety profile, two studies reported no significant difference between melatonin and comparators on preoperative and intra-operative hemodynamic variables, 


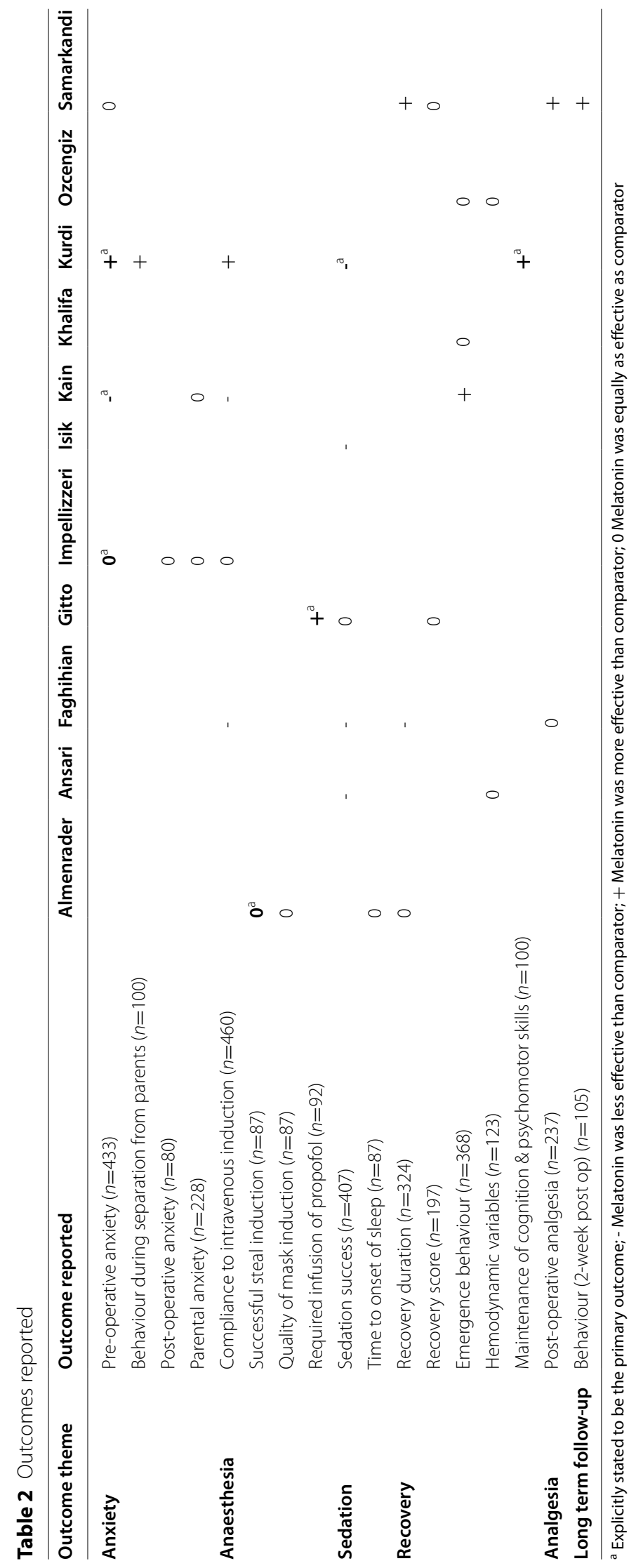


Table 3 Risk of bias assessment of six methodological domains as per the Cochrane risk-of-bias tool (v2.0)

\begin{tabular}{|c|c|c|c|c|c|c|c|}
\hline & $\begin{array}{l}\text { Random } \\
\text { sequence } \\
\text { generation } \\
\text { (selection bias) }\end{array}$ & $\begin{array}{l}\text { Allocation } \\
\text { concealment } \\
\text { (selection bias) }\end{array}$ & $\begin{array}{l}\text { Blinding of } \\
\text { participants } \\
\text { (performance } \\
\text { bias) }\end{array}$ & $\begin{array}{l}\text { Blinding of IMP } \\
\text { administrators } \\
\text { (performance } \\
\text { bias) }\end{array}$ & $\begin{array}{l}\text { Blinding of } \\
\text { outcome } \\
\text { assessment } \\
\text { (detection bias) }\end{array}$ & $\begin{array}{l}\text { Incomplete } \\
\text { outcome data } \\
\text { (attrition bias) }\end{array}$ & $\begin{array}{l}\text { Selective } \\
\text { reporting } \\
\text { (reporting bias) }\end{array}$ \\
\hline $\begin{array}{l}\text { Almenrader } \\
\text { (2013) }\end{array}$ & + & + & - & - & + & + & + \\
\hline Ansari (2018) & + & / & + & - & + & + & + \\
\hline Faghihian (2018) & + & + & + & / & + & / & + \\
\hline Gitto (2016) & + & + & + & + & + & / & + \\
\hline $\begin{array}{l}\text { Impellizzeri } \\
\text { (2017) }\end{array}$ & + & + & + & + & + & + & + \\
\hline Isik (2008) & + & / & + & / & + & + & + \\
\hline Kain (2009) & + & + & + & + & + & + & + \\
\hline Khalifa (2013) & + & + & + & / & + & + & + \\
\hline Kurdi (2016) & + & + & + & - & + & + & + \\
\hline Ozcengiz (2011) & + & + & + & / & + & + & + \\
\hline $\begin{array}{l}\text { Samarkandi } \\
\text { (2005) }\end{array}$ & + & + & + & + & + & + & + \\
\hline
\end{tabular}

+ Low risk of bias; / unspecified; - High risk of bias

including heart rate, electrocardiogram, blood pressure and oxygen saturation [26, 28]. Kurdi et al. assessed the effect of melatonin on cognitive and psychomotor function and found no impact, whereas the comparator, midazolam was significantly associated with cognition and psychomotor dysfunction [25]. The evidence supports melatonin's safety profile in children.

\section{Discussion}

\section{Summary of evidence}

This review identified eleven studies conducted in the pre-operative paediatric setting comparing melatonin with alternative pre-medications. The wide variability in dosing of melatonin, comparators, outcomes and outcome measures used in each study, and inconsistent a priori sample size calculation has limited the ability to draw any definitive conclusion to support or refute melatonin's use as a pre-operative anxiolytic medication. The conflicting results between studies might be in part due to inconsistencies in population, and the dose/formulation of melatonin used. Most studies (eight/eleven) did not study a specifically anxious population. This might reflect a range of practices between different healthcare settings. Within the UK, anaesthetists make the clinical decision on a case-by-case basis as to whether a child should receive a pre-medication for anxiety, whereas in other health care settings routine pre-medication is often standard.

Evidence to support reduction of anxiety and improvement/equivalence of anaesthetic success is conflicting. Four/five included studies indicate that melatonin has a reduced sedative effect compared to comparator [18, $19,25,28]$. No study found melatonin to be inferior on recovery-associated outcomes [21, 23, 24, 26, 27], including the longer-term outcome, reduced sleep disturbance at 2 weeks post-operatively [27]. Kurdi et al. identified a dose-dependent effect of melatonin for alleviating preoperative anxiety in children, with higher doses being more effective [25]. Kain et al. identified a dose-dependent effect of melatonin on improving emergence delirium [23]. These results are consistent with a systematic review and meta-analysis of emergence agitation in children who underwent general anaesthesia, concluding melatonin premedication to be effective in preventing emergence agitation, with increased dose significantly correlating with effect [43]. Improvement in recovery outcomes has potential impact of reduced resource use, faster anaesthetic turnaround times, and improved patient and carer centred outcomes e.g. reduced sedation and emergence dysphoria, and improved compliance.

The review confirms melatonin's excellent safety profile as a pre-medication in children, with very few adverse events recorded and attributable to melatonin. Midazolam, a frequent comparator in the included studies, is a benzodiazepine and is an effective paediatric pre-operative medication [44]. Midazolam has been associated with some adverse effects including sedation and delayed post-operative recovery $[45,46]$. Further concerns include the potential for respiratory suppression [47], and unpredictable effects which may result in agitation rather than anxiolysis, particularly in children with additional needs [48]. The NPSA 2008 rapid 
response document highlights the risk of overdose associated with bolus dosing of midazolam in adults [49], so it is reasonable to also identify alternative pre-medications for the paediatric setting. An alternative premedication, clonidine, has also been associated with adverse events including bradycardia, hypotension and prolonged recovery [50].

\section{Limitations}

Inclusion of a small number of studies overall (total 1047 patients), with large degrees of heterogeneity, preclude a network meta-analysis, therefore studies cannot be combined. The small sample sizes of included studies ( $\max n=148)$, also suggests that the available evidence may not be sufficiently powered to detect a desired magnitude of treatment effect. Examination of the sample size justifications in the studies confirmed this, with nine studies failing to provide a complete justification for the sample size used. This ranged from providing no details on the sample size calculation [19, $21,28]$ to failing to justify the treatment effect to be tested [18, 22, 24-27].

There is clinical difficulty, especially in children, to differentiate between sedation and anxiolysis. The natural sleep-inducing properties of melatonin can produce apparent sedation, enabling anaesthetic induction, without knowing whether sufficient anxiolysis has been obtained. This may have confounded findings in included studies and should be a consideration of future designs to include specifically anxious populations.

\section{Implications for practice/future research}

For future trials, consideration should be given as to whether superiority trial designs are appropriate. The included studies appeared to be designed to test a superiority of treatment effect, whereas for many outcomes superiority of melatonin might not be necessary. A non-inferiority trial design, where an agreed difference in treatment effect is considered not clinically significant, might be preferable if sufficient improvements in other outcomes such as adverse effects or faster anaesthetic turnarounds and reduced resource use, can be demonstrated.

Inconsistencies regarding dose, administration timing, and formulation, outline a need for future trials and pharmacokinetic studies to produce clearer guidance regarding the optimal administration of melatonin as a paediatric pre-medication. Studies within this review suggest that melatonin is more effective at higher doses of 0.5 to $0.75 \mathrm{mg} / \mathrm{kg}$ [25], and less effective at lower doses [23]. Melatonin administration time ranged from $30-60 \mathrm{~min}$ prior to induction (no timing windows reported). This administration schedule is generally consistent with a recent systematic review of the pharmacokinetics of melatonin in adults, which reported the time to maximum melatonin concentration following oral immediate release formulation as 50 min [51].

Included studies trialled varied forms of melatonin. Omission of the trade name/manufacturer of melatonin from a number of studies raises concerns over the quality assurance of the active ingredient trialled. Future trials should use melatonin produced under Good Manufacturing Practice (GMP) [52].

\section{Conclusion}

This systematic review details the current evidence for the use of pre-operative melatonin in children. There is a clear need for more rigorous, larger scale randomised controlled trials to assess the effectiveness of melatonin as a pre-operative medication. Future studies should use a quality assured melatonin product, consider the outcomes to be studied and the statistical design; including a properly justified sample size. The authors of this review are currently involved in a UK wide large, non-inferiority, randomised controlled trial of 624 anxious children aged 5-14, which will try to determine whether melatonin is efficacious in this setting [53].

\section{Abbreviations \\ AE: Adverse Event; FBS: Frankl Behavioural Scale; GMP: Good Manufactur- ing Practice; ICC: Induction Compliance Checklist; PROSPERO : International prospective register of systematic reviews; IV: Intravenous; IB: Investigator Bro- chure; MHRA: Medicines and Healthcare products Regulatory Agency; mYPAS: Modified Yale Pre-operative Anxiety Scale; NHS: National Health Service; OSS: Observers Sedation Scale; PHBQ: Post-Hospital Behaviour Questionnaire; PRISMA : Preferred Reporting Items for Systematic Reviews and Meta-Analyses; RSS: Ramsay Sedation Scale; RCT: Randomised Controlled Trial; RoB: Risk of Bias; STAI: Spielberger State-Trait Anxiety Inventory; STAI-C : Spielberger State- Trait Anxiety Inventory for Children; SmPC: Summary of Product Characteris- tics; UMSS: University of Michigan sedation scale.}

\section{Supplementary Information}

The online version contains supplementary material available at https://doi. org/10.1186/s12887-022-03149-w.

Additional file 1. Electronic search strategy

Acknowledgements

The authors would like to acknowledge the contributions of two medical statisticians, Nikki Totton and Esther Herbert, at the University of Sheffield for their support with reviewing specific aspects of trial design of included studies.

Authors' contributions

The systematic review was first conceived by DP. Study was designed by DP, AT and KM. Searches were performed by KM. Analysis of data was performed by 
KM, AT and DP. KM drafted the manuscript. All authors were involved in critical review of the article. All authors read and approved the final manuscript.

\section{Funding}

Not Applicable.

\section{Availability of data and materials}

The datasets used and/or analysed during the current study are available from the corresponding author on reasonable request.

Additional file 1 includes all search strategies.

\section{Declarations}

\section{Ethics approval and consent to participate}

Not Applicable.

\section{Consent for publication}

Not Applicable.

\section{Competing interests}

The authors declare that they have no competing interests.

\section{Author details}

${ }^{1}$ School of Health and Related Research, University of Sheffield, Sheffield, UK. ${ }^{2}$ School of Clinical Dentistry, University of Sheffield, Sheffield, UK. ${ }^{3}$ Department of Applied Health Research, University College London, London, UK.

\section{Received: 9 June 2021 Accepted: 2 February 2022}

Published online: 24 February 2022

\section{References}

1. Gitto E, Marseglia L, Manti S, D’Angelo G, Barberi I, Salpietro C, et al. Protective role of melatonin in neonatal diseases. Oxid Med Cell Longev. 2013;2013:980374 [cited 2019 Aug 20];Available from: http://www.ncbi. nlm.nih.gov/pubmed/24349616.

2. Chua HM, Richer NH, Swedrowska M, Ingham S, Tomlin S, Forbes B. Dissolution of intact, divided and crushed circadin tablets: prolonged vs. immediate release of melatonin. Pharmaceutics. 2016;8(1) Jan 7 [cited 2019 Jun 25]. Available from: http://www.ncbi.nlm.nih.gov/pubmed/ 26751472.

3. Marseglia L, D’Angelo G, Manti S, Aversa S, Arrigo T, Reiter RJ, et al. Analgesic, anxiolytic and anaesthetic effects of melatonin: new potential uses in pediatrics. Int J Mol Sci [Internet]. 2015;16(1):1209-20 Available from: https://www.ncbi.nlm.nih.gov/pubmed/25569095.

4. Pokharel K, Tripathi M, Gupta PK, Bhattarai B, Khatiwada S, Subedi A. Premedication with oral alprazolam and melatonin combination: A comparison with either alone - A randomized controlled factorial trial. Biomed Res Int. 2014; Epub 2014 Jan 12 Available from: https://www.ncbi.nlm. nih.gov/pmc/articles/PMC3913512/.

5. Khezri M, Merate $H$. The effects of melatonin on anxiety and pain scores of patients, intraocular pressure, and operating conditions during cataract surgery under topical anesthesia. Indian J Ophthalmol. 2013;61(7):319 Available from: http://www.ijo.in/text.asp?2013/61/7/319/ 99637.

6. Ismail SA, Mowafi HA. Melatonin provides anxiolysis, enhances analgesia, decreases intraocular pressure, and promotes better operating conditions during cataract surgery under topical anesthesia. Anesth Analg. 2009;108(4):1146-51.

7. Mowafi HA, Ismail SA. Melatonin improves tourniquet tolerance and enhances postoperative analgesia in patients receiving intravenous regional anesthesia. Anesth Analg. 2008;107(4):1422-6.

8. Caumo W, Torres F, Moreira NL, Auzani JAS, Monteiro CA, Londero G, et al. The clinical impact of preoperative melatonin on postoperative outcomes in patients undergoing abdominal hysterectomy. Anesth Analg. 2007; 105(5):1263-71.

9. Naguib M, Samarkandi AH. Premedication with melatonin: A doubleblind, placebo-controlled comparison with midazolam. Br J Anaesth
[Internet]. 1999;82(6):875-80. Available from:. https://doi.org/10.1093/bja/ 82.6.875.

10. Naguib M, Samarkandi AH. The comparative dose-response effects of melatonin and midazolam for premedication of adult patients: a doubleblinded, placebo-controlled study. Anesth Analg. 2000;91:473-9.

11. Andersen LPH, Werner MU, Rosenberg J, Gögenur I. A systematic review of peri-operative melatonin. Anaesthesia. 2014;69(10):1163-71.

12. Yousaf F, Seet E, Venkatraghavan L, Abrishami A, Chung F. Efficacy and safety of melatonin as an anxiolytic and analgesic in the perioperative period: A qualitative systematic review of randomized trials. Anesthesiology. 2010;113(4):968-76.

13. Hansen M, Halladin N, Rosenberg J, Gögenur I, Møller A. Melatonin for pre- and postoperative anxiety in adults (Review). Cochrane Database Syst Rev. 2015;CD009861(4). Epub Apr2015 Available from: https://www. ncbi.nlm.nih.gov/pmc/articles/PMC6464333/

14. Seabra MLV, Bignotto M, Pinto LR, Tufik S. Randomized, double-blind clinical trial, controlled with placebo, of the toxicology of chronic melatonin treatment. J Pineal Res. 2000;29:193-200.

15. BNF: British National Formulary - NICE [Internet]. NICE; 2019 [cited 2019 Jun 25]. Available from: https://bnf.nice.org.uk/treatment-summary/premedication-and-peri-operative-drugs.html

16. Johnson K, Page A, Williams H, Wassemer E, Whitehouse W. The use of melatonin as an alternative to sedation in uncooperative children undergoing an MRI examination. Clin Radiol. 2002;57(6):502-6 [cited 2019 Nov 22]Available from: https://www.sciencedirect.com/science/article/pii/ S0009926001909237.

17. Impellizzeri P, Vinci E, Gugliandolo MC, Cuzzocrea F, Larcan R, Russo $\mathrm{T}$, et al. Premedication with melatonin vs midazolam: efficacy on anxiety and compliance in paediatric surgical patients. Eur J Pediatr. 2017;176(7):947-53 [cited 2018 Jul 11]; Available from: http://www.ncbi. nlm.nih.gov/pubmed/28540436.

18. Isik B, Baygin Ö, Bodur H, Baygin O, Bodur H, Baygin Ö, et al. Premedication with melatonin vs midazolam in anxious children. Paediatr Anaesth. 2008;18(7):635-41 Available from: https://www.ncbi.nlm.nih.gov/ pubmed/18616492.

19. Faghihian R, Eshghi A, Faghihian H, Kaviani N. Comparison of Oral Melatonin and Midazolam as Premedication in Children Undergoing General Anesthesia for Dental Treatment. Anesthesiol pain Med. 2018;8(2):e64236 Apr [cited 2018 Nov 22]. Available from: http://www.ncbi.nlm.nih.gov/ pubmed/30009151.

20. Almenrader N, Haiberger R, Passariello M. Steal induction in preschool children: is melatonin as good as clonidine? A prospective, randomized study. Paediatr Anaesth. 2013;23(4):328-33 Available from: http://ovidsp. ovid.com/ovidweb.cgi?T =JS\&PAGE=reference \&D=emed $15 \& N E W S=N \&$ $\mathrm{AN}=52379654$

21. Gitto E, Marseglia L, D'Angelo G, Manti S, Crisafi C, Montalto AS, et al. Melatonin versus midazolam premedication in children undergoing surgery: a pilot study. J Paediatr Child Health. 2016;52(3):291-5 Available from: http://cochranelibrary-wiley.com/o/cochrane/clcentral/articles/ 823/CN-01153823/frame.html.

22. Impellizzeri P, Vinci E, Gugliandolo MC, Cuzzocrea F, Larcan R, Russo $T$, et al. Premedication with melatonin vs midazolam: efficacy on anxiety and compliance in paediatric surgical patients. Eur J Pediatr. 2017;176(7):947-53 Available from: http://cochranelibrary-wiley.com/o/ cochrane/clcentral/articles/560/CN-01458560/frame.html.

23. Kain ZN, Maclaren JE, Herrmann L, Mayes L, Rosenbaum A, Hata J, et al. Preoperative melatonin and its effects on induction and emergence in children undergoing anesthesia and surgery. Anesthesiology. 2009;111(1):44-9 Available from: http://ovidsp.ovid.com/ovidweb.cgi?T= JS\&PAGE $=$ reference $\& D=$ emed $12 \& N E W S=N \& A N=354870011$.

24. OSM K, AAM H. Melatonin, ketamine and their combination in half doses for management of sevoflurane agitation in children undergoing adenotonsillectomy Production and hosting by Elsevier Egyptian Society of Anesthesiologists. Egypt J Anaesth. 2013;29:337-41. [cited 2018 Nov 22];Available from. https://doi.org/10.1016/j.egja.2013.05.006.

25. Kurdi MS, Muthukalai SP. A comparison of the effect of two doses of oral melatonin with oral midazolam and placebo on pre-operative anxiety, cognition and psychomotor function in children: A randomised doubleblind study. Indian J Anaesth. 2016;60(10):744-50 Available from: http:// www.ijaweb.org/temp/IndianJAnaesth6010744-7200499_200004.pdf. 
26. Ozcengiz D, Gunes Y, Ozmete O. Oral melatonin, dexmedetomidine, and midazolam for prevention of postoperative agitation in children. $\rfloor$ Anesth. 2011;25(2):184-8 Available from: http://ovidsp.ovid.com/ovidw eb.cgi?T $=J S \& P A G E=$ reference\&D $=$ emed $13 \& N E W S=N \& A N=51280992$.

27. Samarkandi A, Naguib M, Riad W, Thalaj A, Alotibi W, Aldammas F, et al. Melatonin vs. midazolam premedication in children: a double-blind, placebo-controlled study. Eur J Anaesthesiol. 2005;22(3):189-96 Available from: http://ovidsp.ovid.com/ovidweb.cgi?T=JS\&PAGE=reference\&D= emed10\&NEWS $=$ N\&AN $=40694732$.

28. Ansari G, Fathi M, Ghajari MF, Bargrizan M, Eghbali A. Oral Melatonin Versus Midazolam as Premedication for Intravenous Sedation in Pediatric Dental Patients. J Dent (Tehran). 2018;15(5):317-24.

29. Moher D, Liberati A, Tetzlaff J, Altman DG, PRISMA Group. Preferred Reporting Items for Systematic Reviews and Meta-Analyses: The PRISMA Statement. PLoS Med. 2009;6(7):-e1000097 [cited 2018 Aug 13]. Available from: http://www.ncbi.nlm.nih.gov/pubmed/19621072.

30. Singh J. Mendeley: A free research management tool for desktop and web. J Pharmacol Pharmacother. 2010;1(1):62-3 [cited 2019 Jun 25]. Available from: http://www.ncbi.nlm.nih.gov/pubmed/21808593.

31. Higgins J, Sterne J, Savović J, Page M, Hróbjartsson A, Boutron I, et al. A revised tool for assessing risk of bias in randomized trials. Cochrane Database Syst Rev. 2016;10(Suppl 1):29-31.

32. FrankI SN, Shire FR, Fogels HR. Should the parent remain with the child in the dental operatory? J Dent Child. 1962;29(2):150-62.

33. Kain ZN, Mayes LC, Cicchetti DV, Caramico LA, Spieker M, Nygren MM, et al. Measurement tool for preoperative anxiety in young children: The yale preoperative anxiety scale. Child Neuropsychol. 1995;1(3):203-10 Dec [cited 2019 Jan 3. Available from: http://www.tandfonline.com/doi/ abs/10.1080/09297049508400225

34. Spielberger CD. Manual for the State-Trait Anxiety Inventory: STAI (Form Y). Palo Alto: CA Consult Psychol Press; 1983

35. Ramsay MAE, Savege TM, Simpson BRJ, Goodwin R. Controlled Sedation with Alphaxalone-Alphadolone. BMJ. 1974;2(5920):656-9 [cited 2019 Jun 26];Available from: http://www.ncbi.nlm.nih.gov/pubmed/4835444.

36. Mason KP. Pediatric Sedation Outside of the Operating Room: A Multispecialty International Collaboration. 2nd ed. Mason KP, editor. New York: Springer; 2012.

37. Houpt MI, Weiss NJ, Koenigsberg SR, Desjardins PJ. Comparison of chloral hydrate with and without promethazine in the sedation of young children. Pediatr Dent. 1985;7(1):41-6 Mar [cited 2019 Jun 26]. Available from: http://www.ncbi.nlm.nih.gov/pubmed/3857559.

38. Malviya S, Voepel-Lewis T, Tait AR, Merkel S, Tremper K, Naughton N. Depth of sedation in children undergoing computed tomography: validity and reliability of the University of Michigan Sedation Scale (UMSS). Br J Anaesth. 2002;88(2):241-5 [cited 2019 Jun 26]. Available from: http:// www.ncbi.nlm.nih.gov/pubmed/11878656.

39. Keegan NJ, Yudkowitz FS, Bodian CA. Determination of the reliability of three scoring systems to evaluate children after general anesthesia. Anaesthesia. 1995;50(3):200-2 [cited 2019 Jun 26]. Available from: http:// www.ncbi.nlm.nih.gov/pubmed/7717482.

40. Aldrete JA, Kroulik D. A postanesthetic recovery score. Anesth Analg. 1970;49(6):924-34 [cited 2019 Apr 18]. Available from: http://www.ncbi. nlm.nih.gov/pubmed/5534693.

41. Aldrete JA. The post-anesthesia recovery score revisited. J Clin Anesth. 1995;7(1):89-91 Feb 1 [cited 2019 Apr 18];. Available from: https://www. sciencedirect.com/science/article/pii/095281809400001K?via\%3Dihub.

42. Jenkins BN, Kain ZN, Kaplan SH, Stevenson RS, Mayes LC, Guadarrama $J$, et al. Revisiting a measure of child postoperative recovery: Development of the Post Hospitalization Behavior Questionnaire for Ambulatory Surgery. Paediatr Anaesth. 2015;25(7):738-45.

43. Mihara T, Nakamura N, Ka K, Oba MS, Goto T. Effects of melatonin premedication to prevent emergence agitation after general anaesthesia in children. Eur J Anaesthesiol. 2015; Jul [cited 2019 Aug 20];1. Available from: http://content.wkhealth.com/linkback/openurl?sid=WKPTLP:landi ngpage\&an=00003643-900000000-98905.

44. McCluskey A, Meakin GH. Oral administration of midazolam as a premedicant for paediatric day-case anaesthesia. Anaesthesia. 1994;49(9):782-5 Available from: https://www.ncbi.nlm.nih.gov/pubmed/7978134.

45. Cray SH, Dixon JL, Heard CM, Selsby DS. Oral midazolam premedication for paediatric day case patients. Paediatr Anaesth. 1996;6(4):265-70 Available from: https://www.ncbi.nlm.nih.gov/pubmed/8827741.
46. Viitanen $\mathrm{H}$, Annila P Viitanen M, Tarkkila P Premedication with midazolam delays recovery after ambulatory sevoflurane anesthesia in children. Anesth Analg. 1999;89(1):75-9 Available from: https://www.ncbi.nlm.nih. gov/pubmed/10389782.

47. Sievers TD, Yee JD, Foley ME, Blanding PJ, Berde CB. Midazolam for conscious sedation during pediatric oncology procedures: safety and recovery parameters. Pediatrics. 1991;88(6):1172-9 Available from: https://www.ncbi.nlm.nih.gov/pubmed/1956734.

48. Maeda S, Tomoyasu Y, Higuchi H, Mori T, Egusa M, Miyawaki T. Midazolam is associated with delay in recovery and agitation after ambulatory general anesthesia for dental treatment in patients with disabilities: a retrospective cohort study. J Oral Maxillofac Surg. 2012;70(6):1315-20 Available from: https://www.ncbi.nlm.nih.gov/pubmed/22381698.

49. NHS National Patient Safety Agency. Rapid Response Report: NPSA/2008/ RRR011: Reducing risk of overdose with midazolam injection in adults. NHS. 2008.

50. Tazeroualti N, De Groote F, De Hert S, De Villé A, Dierick A, Van der Linden P. Oral clonidine vs midazolam in the prevention of sevoflurane-induced agitation in children. A prospective, randomized, controlled trial †. $\mathrm{Br} J$ Anaesth. 2007;98(5):667-71 Apr [cited 2019 Jan 3]Available from: http:// www.ncbi.nlm.nih.gov/pubmed/17416907.

51. Harpsøe NG, Andersen LP, Gögenur I, Rosenberg J. Clinical pharmacokinetics of melatonin: a systematic review. Eur J Clin Pharmacol. 2015;71(8):901-9 Available from: https://www.ncbi.nlm.nih.gov/pubmed/ 26008214.

52. COMMISSION DIRECTIVE 2003/94/EC of 8 October 2003 laying down the principles and guidelines of good manufacturing practice in respect of medicinal products for human use and investigational medicinal products for human use (Text with EEA relevance) [Internet]. [cited 2019 Jun 7]. Available from: https://eur-lex.europa.eu/LexUriServ/LexUriServ. do?uri=OJ:L:2003:262:0022:0026:en: PDF.

53. ISRCTN - ISRCTN18296119: Melatonin for Anxiety prior to General anaesthesia In Children (MAGIC) [Internet]. [cited 2019 Jun 7]. Available from: http://www.isrctn.com/ISRCTN18296119

\section{Publisher's Note}

Springer Nature remains neutral with regard to jurisdictional claims in published maps and institutional affiliations.

Ready to submit your research? Choose BMC and benefit from

- fast, convenient online submission

- thorough peer review by experienced researchers in your field

- rapid publication on acceptance

- support for research data, including large and complex data types

- gold Open Access which fosters wider collaboration and increased citations

- maximum visibility for your research: over $100 \mathrm{M}$ website views per year

At BMC, research is always in progress.

Learn more biomedcentral.com/submissions 